\title{
Contaminación por nitratos y fosfatos provenientes de la actividad agrícola en la cuenca baja del río Mayo en el estado de Sonora, México Pollution by nitrates and phosphates from agricultural activity in the low basin of the Mayo river in the state of Sonora, Mexico
}

\author{
Isaac Shamir Rojas-Rodríguez ${ }^{1(\mathbb{D})}$, Manuel Arturo Coronado-García ${ }^{2:}$ (D), \\ Sergio Ramón Rossetti-López ${ }^{1}{ }^{\circledR}$ y Félix Alfredo Beltrán-Morales ${ }^{2} \odot$
}

\footnotetext{
${ }^{1}$ Universidad de Sonora. Departamento de Administración. Edificio 10G, Contabilidad y Administración, Blvd. Luis Encinas y Rosales s/n, Col. Centro. 83000 Hermosillo, Sonora, México.

${ }^{2}$ Universidad Autónoma de Baja California Sur. Carretera al Sur km 5.5, Colonia El Mezquitito. 23080 La Paz, B. C. S., México.

*Autor para correspondencia (mcoronado@uabcs.mx)
}

\section{RESUMEN}

La contaminación de los cuerpos de agua dentro de las cuencas hidrológicas ha cobrado relevancia en la última década. Se señala a la agricultura como la principal actividad antropogénica generadora de este fenómeno de contaminación. Los estudios especializados, definen a este tipo de contaminantes como "contaminación difusa" debido a la forma en que se distribuye dentro de las cuencas. Entre las principales substancias reportadas provenientes de la agricultura se encuentran nitratos y fosfatos, como remanentes de los procesos de fertilización. El objetivo de este trabajo fue caracterizar el aporte de nitratos y fosfatos provenientes de la agricultura en la cuenca baja del río Mayo en el estado de Sonora, México; con la finalidad de comparar los escenarios línea base y el escenario de contaminación que genera la producción de trigo en la región. La hipótesis propone que el aporte de nitratos y fosfatos proveniente de la agricultura rebasa lo establecido por un escenario línea base. Mediante la aplicación del modelo de simulación hidrológica Soil and Water Assessment Tool (SWAT), fueron simulados, las aportaciones de substancias contaminantes dentro de la cuenca de estudio para el ciclo comprendido entre los años 2000 y 2017. El estudio descriptivo de la información se realizó mediante la comparación de las medias donde, para todos los casos los resultados de la simulación agrícola superaron los parámetros obtenidos en el escenario línea base. Encontrando diferencia estadísticamente

Cita recomendada:

Rojas-Rodríguez, I. S., M. A. Coronado-García, S. R. Rossetti-López y F. A. Beltrán-Morales. 2020. Contaminación por nitratos y fosfatos provenientes de la actividad agrícola en la cuenca baja del río Mayo en el estado de Sonora, México. Terra Latinoamericana 38: 247-256.

DOI: https://doi.org/10.28940/terra.v38i2.642 significativa para los casos de nitrato y dióxido de nitrógeno. Los resultados muestran evidencia que la realización de actividad agrícola dentro de la cuenca hidrológica analizada genera un incremento en las aportaciones de substancias superando el escenario línea base estimado.

Palabras clave: ciclo del agua, cuerpos de agua, hidrología, modelo SWAT, simulación.

\section{SUMMARY}

The pollution of water bodies within watersheds has gained relevance in the last decade. Agriculture is designated as the main anthropogenic activity that generates this pollution phenomenon. Specialized studies define this type of pollutants as "diffuse pollution" due to the way that they are distributed within the basins. Among the main substances reported from agriculture are nitrates and phosphates, as remnants of fertilization processes. The objective of our study was to characterize the contribution of nitrates and phosphates from agriculture in the Lower Basin of the Mayo River in the State of Sonora, Mexico, with the purpose of comparing the baseline scenarios and the pollution scenario derived from wheat agriculture in the region. In this research we hypothesize that the contribution of nitrates and phosphates from agriculture exceeds what is established in the baseline scenario. By applying the hydrological simulation model Soil and Water Assessment Tool (SWAT), we simulated

Recibido: 17 de septiembre de 2019. Aceptado: 19 de octubre de 2019. Publicado en Terra Latinoamericana 38: 247-256. 
the contributions of polluting substances within the basin under study for the period of 2000 to 2017. The descriptive study was performed by comparing means where, for all cases, the results of the agricultural simulation exceeded the parameters obtained in the baseline scenario. Significant differences were found for nitrate and nitrogen dioxide. The results show evidence of the fact that the agricultural activity within the hydrological basin under study generates an increase in the contributions of substances exceeding the estimated baseline scenario.

Index words: water cycle, water bodies, hydrology, SWAT model, simulation.

\section{INTRODUCCIÓN}

Diversos estudios han confirmado que, entre los principales precursores de daños a los cuerpos de agua se encuentran las actividades agrícolas, generando su detrimento gracias a la contaminación por pesticidas y fertilizantes, provocando alteraciones a nivel de cuencas hidrológicas (Smith et al., 2017; Ouyang et al., 2019; Reaney et al., 2019). Los estudios realizados en la "Evaluación de los Ecosistemas del Milenio" (Reid et al., 2005) vincularon el efecto degenerativo de la actividad agropecuaria sobre los recursos naturales a través de la escorrentía pluvial de substancias contaminantes en cuencas hidrológicas. El catálogo de elementos que escurren en las cuencas se compone principalmente de nitrógeno y fósforo; además de substancias orgánicas e inorgánicas depositadas en diferentes cuerpos de agua y cuyo comportamiento resulta ser aleatorio, asimétrico e incierto, lo que las define como fuentes de contaminación difusa.(Zhou et al., 2012; Zhang et al., 2015).

La contaminación proveniente de la actividad agrícola o contaminación difusa, es difícil de detectar, medir y controlar; porque involucra relaciones complejas que se suscitan dentro de las cuentas hidrológicas. Debido a que la contaminación difusa se transporta a través de la escorrentía, está naturalmente asociada con precipitación y se asocia esencialmente con el uso y cobertura del suelo. Factores como: precipitaciones, temperaturas, uso del suelo, tipo de suelo, orografía, vegetación, entre otras, en combinación con las características de las zonas agrícolas insertas en las cuencas hidrológicas, resultan indefectibles para estimar el flujo de contaminación difusa hacia los lugares de deposición en estos espacios. El estudio de los ciclos biogeoquímicos que comprenden los territorios conformados por las cuencas hidrológicas conduce a la creación de su tipología, con base en la cantidad de contaminantes que pueden transportar a través de ellas (Zhang et al., 2015; Xiao et al., 2017; Oliveira et al., 2017).

Estudios señalan que, frente a la carencia de mecanismos para la creación de políticas en referencia a la agricultura y el medio ambiente, se pone de manifiesto la importancia de realizar análisis espaciotemporales a nivel cuenca para entender el origen y desplazamiento de la contaminación. Para tal propósito, advierten la pertinencia de incorporar enfoques que aborden el fenómeno de la contaminación desde una triple dimensión: económica, social y ambiental (Turner et al., 2006; Galloway et al., 2008).

Para el estudio de la dispersión de contaminantes a nivel de cuenta hidrológica, destaca el uso del modelo SWAT (Soil and Water Assessment Tool, Herramienta de Evaluación de Suelos y Aguas). Esta herramienta es capaz de reproducir el modelado hidrológico de diversos territorios donde inciden diferentes actividades productivas. El modelo SWAT, se ha convertido en una de las herramientas más utilizadas en los últimos años para el estudio de la contaminación proveniente de la agricultura, debido a su eficiencia para simular el impacto que causan actividades dentro de la agricultura, como el manejo de los suelos, tipo de riego, tipo de cultivos, cosechas, aplicación de pesticidas y su fertilización (Liu et al., 2013; Maharjan et al., 2016; Özcan et al., 2017).

En países como México, la creación de políticas efectivas que atiendan los impactos de la agricultura en el medio ambiente se complica, ante la carencia de mecanismos adecuados para la medición de la contaminación difusa dentro delas cuencas hidrológicas. El estudio de la interacción de los componentes de los ecosistemas con las actividades económicas, puede ser abordado de manera integral desde el enfoque de cuencas. El análisis interdisciplinario de la interacción del hombre con los ecosistemas, confirma la incidencia de agentes socioeconómicos cuyas actividades productivas comprometen la calidad y cantidad de los recursos naturales. El uso que se le ha dado a los recursos naturales tanto como materias primas como captadores de desechos, alteran la integridad de los ecosistemas reduciendo su utilidad presente y futura (Cotler et al., 2013; Babin et al., 2016). 
En el caso de las cuencas hidrológicas que prestan varios servicios ambientales a las actividades económicas, es preciso determinar la vocación natural de esos territorios a fin de aprovechar de manera sustentable sus recursos naturales. El análisis de los componentes de los ecosistemas y su interacción para propiciar escorrentía en un territorio con una intensa actividad agrícola, lo convierte en una zona propensa a generar afectaciones ambientales en los drenes exorreicos o endorreicos, por el aporte de contaminación difusa. La realización de este análisis requiere del estudio de las interacciones que guardan los diferentes elementos de los ecosistemas con las actividades antropogénicas (Haro y Taddei, 2014).

Tal es el caso de la cuenca baja del río Mayo en el estado de Sonora en México, donde las actividades antropogénicas desarrolladas en este espacio están lideradas por el sector agrícola como el eje central de la economía y la producción de trigo es la actividad agrícola preponderante en la región, al destinarse $70 \%$ de la superficie para este cultivo (SIAP, 2018). Sin embargo, también comprende un importante componente pecuario basado en la cría de becerros de exportación y producción de camarón. Los servicios ambientales de aprovisionamiento que presta esta cuenca la convierten en un ecosistema importante para el desempeño del desarrollo económico de la región (Haro et al., 2015).

Conocer el equilibrio ambiental que prevalece dentro de los ecosistemas es necesario para determinar el impacto de las actividades económicas sobre el estado natural del medio ambiente. Este equilibrio puede ser determinado mediante la realización de estudios de línea base ambiental. La función principal de los estudios de línea base ambiental, es la de recopilar información que pueda ser utilizada para detectar cambios en los ecosistemas. Modelos matemáticos son utilizados para estimar la variabilidad ante la presencia de efectos que alteran el comportamiento normal de los indicadores de referencia. La detección de indicadores sobre del estado que prevalece en el medio ambiente es una acción crítica para la elaboración de programas de monitoreo ambiental. Las actividades antropogénicas requieren de constante monitoreo debido a que son desarrolladas dentro de diversos ecosistemas, los datos de monitoreo de contaminación pueden ser utilizados para desarrollar una evaluación línea base a fin de establecer indicadores ecológicos (Large et al., 2013; Andrews et al., 2015; Linder et al., 2017).
En este trabajo se caracterizó el aporte de nitratos y fosfatos provenientes de la agricultura mediante la aplicación de un modelo de simulación hidrológica en la cuenca baja del río Mayo en el estado de Sonora, México, con la finalidad de generar y comparar los escenarios línea base y el escenario de contaminación que propicia la actividad agrícola de la región.

\section{MATERIALES Y MÉTODOS}

Diseño de la investigación. Se basa en un estudio longitudinal que comprende los años 2000 a 2017.

Zona de estudio. Los municipios suscritos a la cuenca baja del río Mayo, en el estado de Sonora, suman una superficie total de $3224 \mathrm{~km}^{2}$, equivalente a $1.79 \%$ del territorio estatal, los cuales se distribuyen espacialmente de la siguiente forma: Álamos $8.11 \%$, Etchojoa, 37.61\%, Huatabampo 28.68\%, Navojoa $58.79 \%$ y Quiriego $4.55 \%$ (Figura 1). Las principales actividades antropogénicas de orden económico desarrolladas en este espacio están lideradas por el sector agropecuario donde la agricultura es el eje central de la economía (Figura 2). Sin embargo, también comprende un importante componente pecuario basado principalmente en la cría de becerros de exportación. Además de ocupar el primer lugar nacional en la producción semi-intensiva de camarón blanco.

Modelo Soil and Water Assessment Tool (SWAT). En el contexto académico el modelo SWAT constituye una herramienta multicitada. Entre sus características

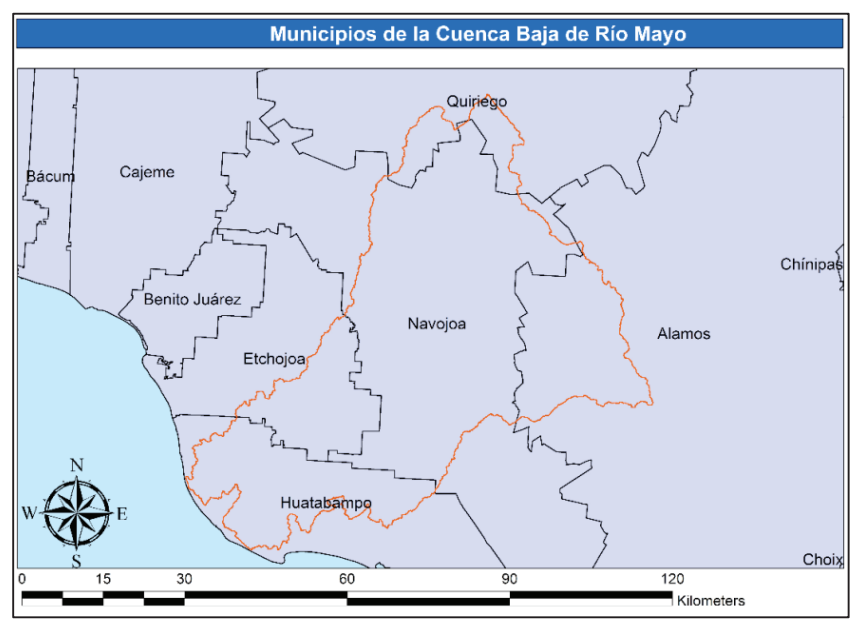

Figura 1. Municipios que conforman la cuenca baja del río Mayo. Fuente: Elaboración propia (2019).

Figure 1. Municipalities conforming the lower basin of the Mayo River. Source: Elaborated by the authors (2019). 


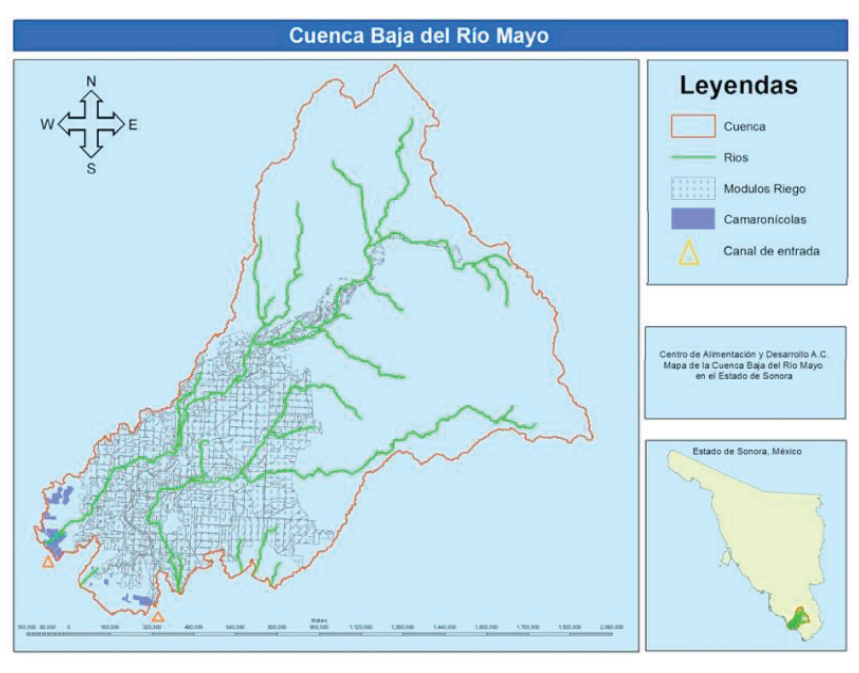

Figura 2. Principales actividades económicas en la cuenca baja del río Mayo en el estado de Sonora. Fuente: Elaboración propia (2019).

Figure 2. Main economic activities in the lower basin of the Mayo River in the state of Sonora. Source: Elaborated by the authors (2019).

está el efectuar: simulaciones dinámicas en diversas temporalidades (diarias, mensuales o anuales), la predicción de impactos específicos como el de uso y manejo del suelo en el agua, así como estimar la sedimentación y rendimientos agrícolas en las cuencas hidrológicas. El modelo -basado en procesos- es capaz de efectuar simulaciones de largos periodos de tiempo. Sus insumos son el clima, precipitación, temperatura, propiedades del suelo, crecimiento de plantas, nutrientes, plaguicidas, y uso del suelo, entre otros. En el SWAT, una cuenca se divide en múltiples subcuencas, que luego se subdividen en Unidades de Respuesta Hidrológica (URH), consistentes en unidades que presentan arreglos homogéneos característicos en uso de la tierra, manejo, topografía y características del suelo. Las URH se representan como un porcentaje del área de la cuenca. El balance hídrico es la fuerza impulsora detrás de todos los procesos en el Modelo porque afecta el crecimiento de las plantas y movimiento de sedimentos, nutrientes, pesticidas y patógenos.

Este modelo puede leer los datos de entrada directamente desde archivos o generar datos simulados en tiempo de ejecución a partir de la información introducida. Los procesos hidrológicos simulados incluyen: Almacenamiento de agua de la vegetación, escurrimiento superficial, infiltración, evapotranspiración, flujo lateral, láminas de drenaje, perfil del suelo, el consumo por medio del bombeo (si existe), retorno de flujo y recarga por filtración desde la superficie, cuerpos de agua, estanques y canales tributarios. Además de modelar el movimiento y la transformación de varias formas de nitrógeno, fósforo, plaguicidas y sedimentos en la cuenca (Neitsch et al., 2009).

El objetivo del modelo es el de reproducir el movimiento del agua subterránea en la zona en estudio. En este trabajo se utilizó el modelo SWAT, como plug in del software ArcGis versión 10.3, cuyos requerimientos de información se muestran en la Figura 3. La ecuación que rige el comportamiento hidrológico estudiado por el modelo es la siguiente Ecuación (1) del modelo hidrológico utilizado.

$S W t=S W 0+\sum_{i=0}^{n}(R d a y-Q \operatorname{sur} f-E a-w s e e p-Q w g)$

Fuente: Neitsch et al. (2009).

donde: SWt es el contenido final de agua en el suelo $\left(\mathrm{mm} \mathrm{H}_{2} \mathrm{O}\right)$, SW0 es el contenido inicial de agua del suelo en un día $\mathrm{i}\left(\mathrm{mm} \mathrm{H}_{2} \mathrm{O}\right)$, $\mathrm{t}$ es el tiempo (días), Rday es la cantidad de precipitación en un día i $\left(\mathrm{mm} \mathrm{H}_{2} \mathrm{O}\right)$, Qsurf es la cantidad de escorrentía de la superficie en un día $\mathrm{i}\left(\mathrm{mm} \mathrm{H}_{2} \mathrm{O}\right)$, Ea es la cantidad de evapotranspiración en día $\mathrm{i}\left(\mathrm{Mm} \mathrm{H}_{2} \mathrm{O}\right)$, Wseep es la cantidad de agua que percola en el perfil del suelo en un día $\mathrm{i}\left(\mathrm{mm} \mathrm{H}_{2} \mathrm{O}\right)$, y Qgw es la cantidad de flujo de retorno en un día $\mathrm{i}\left(\mathrm{mm} \mathrm{H}_{2} \mathrm{O}\right)^{1}$.

Información topográfica. El Continuo de Elevaciones Mexicano 3.0 (CEM 3.0) está conformado por un conjunto de datos ráster del territorio mexicano, dentro del cual se muestra información tridimensional a fin de conocer las diferentes elevaciones de las zonas de estudio (INEGI, 2016).

Uso del suelo. Los datos referentes al uso del suelo de la zona de estudio se obtuvieron de la información generada por CONABIO, la cual contiene datos sobre el uso del suelo de la zona (CONABIO, 2016).

Tipos de suelos. Este conjunto de información muestra el tipo de suelo que se encuentra en el territorio nacional, donde destacan el: Regosol, Litosol y Xerosol. Esta información contiene diferentes datos relacionados a sus fases tanto químicas como físicas,

${ }^{1}$ Extraído de https://swat.tamu.edu/media/46967/swat2005-tutorial-spanish.pdf. 


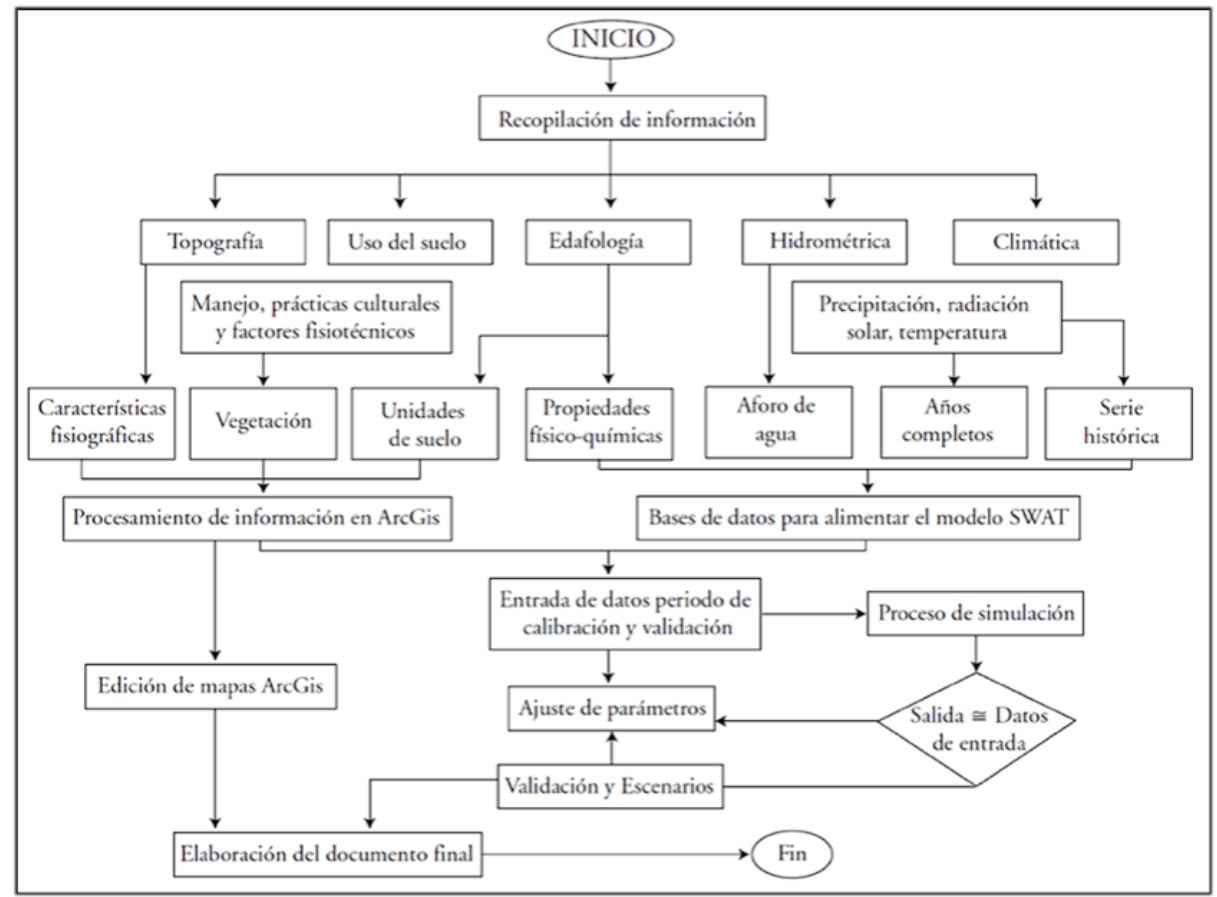

Figura 3. Proceso de simulación para el modelado hidrológico que sigue el SWAT. Fuente: Neitsch et al. (2009).

Figure 3. Simulation process for hydrological modeling followed by SWAT. Source: Neitsch et al. (2009).

textura, permeabilidad entre otras. De este conjunto de datos se tomó solo la información correspondiente a la zona de estudio. (CONABIO, 2016).

Información climatológica. La información referente al clima para la alimentación del modelo se obtuvo de la base de datos del portal Global Weather Data for SWAT (SWAT, 2018). Dentro de la zona de estudio se encuentran 6 estaciones climatológicas de las cuales se obtuvieron datos diarios de precipitación, temperaturas máximas y mínimas correspondientes distintos periodos que van desde 1980 a 2010. Estos datos fueron adaptados dentro de una base de datos para ser ingresados al modelo.

Manejo de cultivos. Los principales municipios que integran la cuenca se encuentran especializados en una agricultura altamente tecnificada, que tiene como soporte la utilización intensiva del recurso hídrico bajo sistemas de riego por gravedad. En este espacio se identifica un área empadronada de 97891 ha en el Distrito de Riego 149, con una superficie irrigable de 92000 hectáreas. El patrón de cultivos es liderado por la producción triguera extendida en 58 . El 22\% de la superficie de siembra disponible, seguida en orden de importancia por el cártamo con $11.15 \%$ de la superficie sembrada (Cuadro 1). La información sobre el manejo del cultivo del trigo se obtuvo del paquete tecnológico de INIFAP (Cuadro 2).

Estudio de línea base. Para llevar a cabo el estudio de línea base, se ingresó en el modelo SWAT la información necesaria, tomando en cuenta que para el uso del suelo no se capturaron los datos relacionados al desarrollo de la actividad agrícola, a fin de obtener los resultados de la escorrentía simulada sin la presencia de zonas agrícolas en la región.

Análisis Estadístico. Se realizó una prueba Chapiro Wilk para muestras menores a 30 , a fin de determinar la normalidad de los datos de los contaminantes nitrógeno orgánico, fósforo orgánico, nitrato, amonio, dióxido de nitrógeno y fósforo mineral obtenidos en la simulación de ambos escenarios. Esta prueba obtuvo significancia estadística, por lo tanto, se concluye que los datos no provienen de una distribución normal. Basados en los resultados anteriores, se procedió a realizar la prueba Wilcoxon para la comparación de las medianas de los contaminantes con un nivel de significancia estadística alfa de 0.05 . 
Cuadro 1. Patrón regional de cultivos.

Table 1. Regional crop pattern.

\begin{tabular}{|c|c|c|c|c|c|c|}
\hline Cultivo & 2012 & 2013 & 2014 & 2015 & 2016 & \\
\hline Trigo & 49018 & 79559 & 82550 & 93798 & 89136 & 78812 \\
\hline Cártamo & 35619 & 4822 & 14708 & 9889 & 10440 & 15097 \\
\hline Sorgo & 14150 & 15109 & 12627 & 10487 & 11878 & 12850 \\
\hline Ajonjolí & 6000 & 6000 & 8268 & 7130 & 5308 & 6541 \\
\hline Papa & 5932 & 4898 & 4764 & 5385 & 5400 & 5275 \\
\hline Maíz & 4887 & 7194 & 3662 & 3849 & 3593 & 4637 \\
\hline Superficie sembrada & 126569 & 127871 & 136431 & 142216 & 143663 & 135350 \\
\hline
\end{tabular}

Fuente: Elaboración propia con base en SIAP, 2017. Source: Elaborated by the authors based on SIAP, 2017.

\section{RESULTADOS Y DISCUSIÓN}

Con los resultados de la aplicación del modelo de simulación, se generaron dos escenarios; uno de línea base, es decir, sin la intrusión de la actividad agrícola dentro de la zona de estudio y otro donde se ingresaron los datos del manejo de cultivo del trigo. Como resultado se presentan los Cuadros 3 y 4, las cuales contienen los datos de los diferentes contaminantes simulados por el modelo SWAT.

En la comparación de medianas por escenarios (línea vs agrícola) destaca el incremento de aportes en el escenario agrícola en las substancias mostradas en los Cuadros 3 y 4 , sin embargo los resultados de la prueba estadística de Wilcoxon, la cual permite realizar el análisis de datos de un experimento de muestras pareadas, se logró determinar que, para los casos de los contaminantes nitrato y dióxido de nitrógeno, los resultados de la simulación agrícola muestran evidencia para establecer que existen diferencias significativas en las medianas de nitrato y dióxido de nitrógeno con un nivel de significación alfa de 0.05 , es decir, los valores de ambos contaminantes superan las aportaciones del escenario agrícola con respecto al escenario línea base. Con lo anterior se rechaza la hipótesis nula $(\mathrm{Ho})$ y se concluye que existe diferencia significativa entre el aporte de contaminantes entre el escenario línea base y el escenario agrícola.

Cuadro 2. Paquete tecnológico para el manejo del trigo.

Table 2. Technological package for wheat management.

\begin{tabular}{lcclc}
\hline Año & Mes & Día & Momento de aplicación & Cantidad \\
\hline & & & Riego & $\mathrm{mm}$ \\
1 & 11 & 1 & Riego pre-siembra & 270 \\
2 & 1 & 4 & Primero & 170 \\
2 & 2 & 3 & Segundo & 160 \\
2 & 2 & 23 & Tercero & 160 \\
& & & Fertilizante & $30 \mathrm{~kg}$ urea \\
1 & 11 & 15 & Pre-siembra & $100 \mathrm{~kg} 11-52-00$ \\
2 & 11 & 15 & Pre-siembra & $100 \mathrm{~kg}$ Anhydrous ammonia \\
2 & 1 & 4 & Primero & $50 \mathrm{~kg}$ Anhydrous ammonia \\
\hline
\end{tabular}

Fuente: Moreno (2019)². Source: Moreno (2019)².

${ }^{2}$ Moreno-Dena, J. M. 2019. Impacto económico del cambio climático en el cultivo del trigo en regiones productoras de Sonora, México. Tesis de doctorado. Moreno Dena Jesús Mario_DDR_2015-2019.pdf. CIAD. Hermosillo, Sonora, México. 
Cuadro 3. Generación de contaminantes simulados para el escenario línea base.

Table 3. Generation of simulated pollutants for the baseline scenario.

\begin{tabular}{|c|c|c|c|c|c|c|}
\hline Año & $\begin{array}{l}\text { Nitrógeno } \\
\text { orgánico }\end{array}$ & Fósforo orgánico & Nitrato & Amonio & $\begin{array}{l}\text { Dióxido de } \\
\text { nitrógeno }\end{array}$ & Fósforo minera \\
\hline & $\ldots$ & $-\ldots$ & 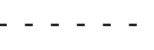 & $\cdots \cdots$ & $\ldots$ & $\ldots-\cdots$ \\
\hline 2000 & 7422.52 & 1172.06 & 5554.96 & 1810.43 & 838.25 & 724.90 \\
\hline 2001 & 3832.32 & 495.12 & 16504.92 & 1980.17 & 986.17 & 2233.06 \\
\hline 2002 & 4565.97 & 555.15 & 46120.27 & 2287.96 & 887.37 & 2015.39 \\
\hline 2003 & 5.61 & 1.78 & 2256.11 & 85.75 & 33.85 & 26.71 \\
\hline 2004 & 4757.16 & 599.34 & 23060.55 & 2285.27 & 1379.89 & 1974.69 \\
\hline 2005 & 1760.22 & 223.16 & 27334.64 & 1033.69 & 395.55 & 841.66 \\
\hline 2006 & 382.91 & 56.08 & 36519.68 & 459.90 & 141.60 & 491.5 \\
\hline 2007 & 1533.27 & 246.59 & 30844.85 & 943.38 & 226.77 & 810.97 \\
\hline 2008 & 48.89 & 8.87 & 4404.88 & 113.31 & 38.79 & 81.55 \\
\hline 2009 & 4534.34 & 654.52 & 65228.98 & 2016.08 & 890.76 & 1715.22 \\
\hline 2010 & 144.85 & 25.09 & 15172.24 & 149.77 & 48.33 & 713.80 \\
\hline 2011 & 52.47 & 8.62 & 4349.64 & 104.83 & 35.79 & 159.37 \\
\hline 2012 & 163.95 & 28.47 & 17538.29 & 223.19 & 76.34 & 174.72 \\
\hline 2013 & 2325.98 & 343.29 & 35777.37 & 1135.45 & 418.91 & 1259.41 \\
\hline 2014 & 2013.61 & 309.84 & 139023.96 & 1196.38 & 370.13 & 851.17 \\
\hline 2015 & 14690.53 & 2265.25 & 62776.38 & 4952.01 & 1286.1 & 3076.56 \\
\hline 2016 & 4678.07 & 760.36 & 5902.10 & 1147.89 & 241.48 & 462.53 \\
\hline 2017 & 1063.87 & 165.56 & 11602.14 & 630.18 & 253.56 & 678.85 \\
\hline Mediana & 1887 & 278 & 2099 & 1085 & 312 & 768 \\
\hline $\begin{array}{l}\text { Normalidad } \\
\text { Valor de P }\end{array}$ & $<0.010$ & $<0.010$ & $<0.010$ & $<0.010$ & 0.03 & $>0.100$ \\
\hline
\end{tabular}

Fuente: Elaboración propia (2019). Source: Elaborated by the authors (2019).

En la actualidad el Sistema de Consulta de Estadísticas Ambientales del INEGI no establece indicadores que describan el impacto ambiental de las actividades agrícolas. Los cuales se pueden generar a partir de resultados como los mostrados en los Cuadros 3 y 4, así como la pertinencia de conocer los lugares de mayor impacto por el proceso de escorrentía que se muestra en la Figura 4, donde destaca la ubicación de los drenes exorreicos de la cuenca.

En la Figura 4 se aprecia el aporte de las salidas o drenes naturales de la cuenca, por medio de los cuales deposita la escorrentía en las aguas costeras, llevando con ellas las cantidades de contaminantes expresados en los Cuadros 3 y 4 . Se observan visibles incrementos en los aportes de contaminantes los años 2004 y 2015. La información generada debe de ser analizada a detalle a fin de determinar sus posibles afectaciones a los ecosistemas que reciben estas aguas y a las actividades económicas que se llevan a cabo en ellos. Los indicadores deben ser generados y evaluados con modelos para desarrollar estándares que cuantifiquen los efectos antropogénicos en el medio ambiente. Las alteraciones en los ecosistemas son resultado del inicio, fin o variación en la intensidad de las actividades humanas, como son las actividades industriales, la agricultura o el cambio climático.

En la revisión bibliográfica destacan trabajos en donde se mencionan los aportes de contaminación difusa por parte de actividades como la ganadería, como el de Madroñero-Palacios y Jiménez-Otárola (2019) y el de González et al. (2019) el cual determina la vulnerabilidad de cuerpos de agua, sin embargo, estos no reportan cuantificación de los aportes de contaminación por las actividades productivas. 
Cuadro 4. Generación de contaminantes simulados para el escenario agrícola.

Table 4. Generation of simulated pollutants for the agricultural scenario.

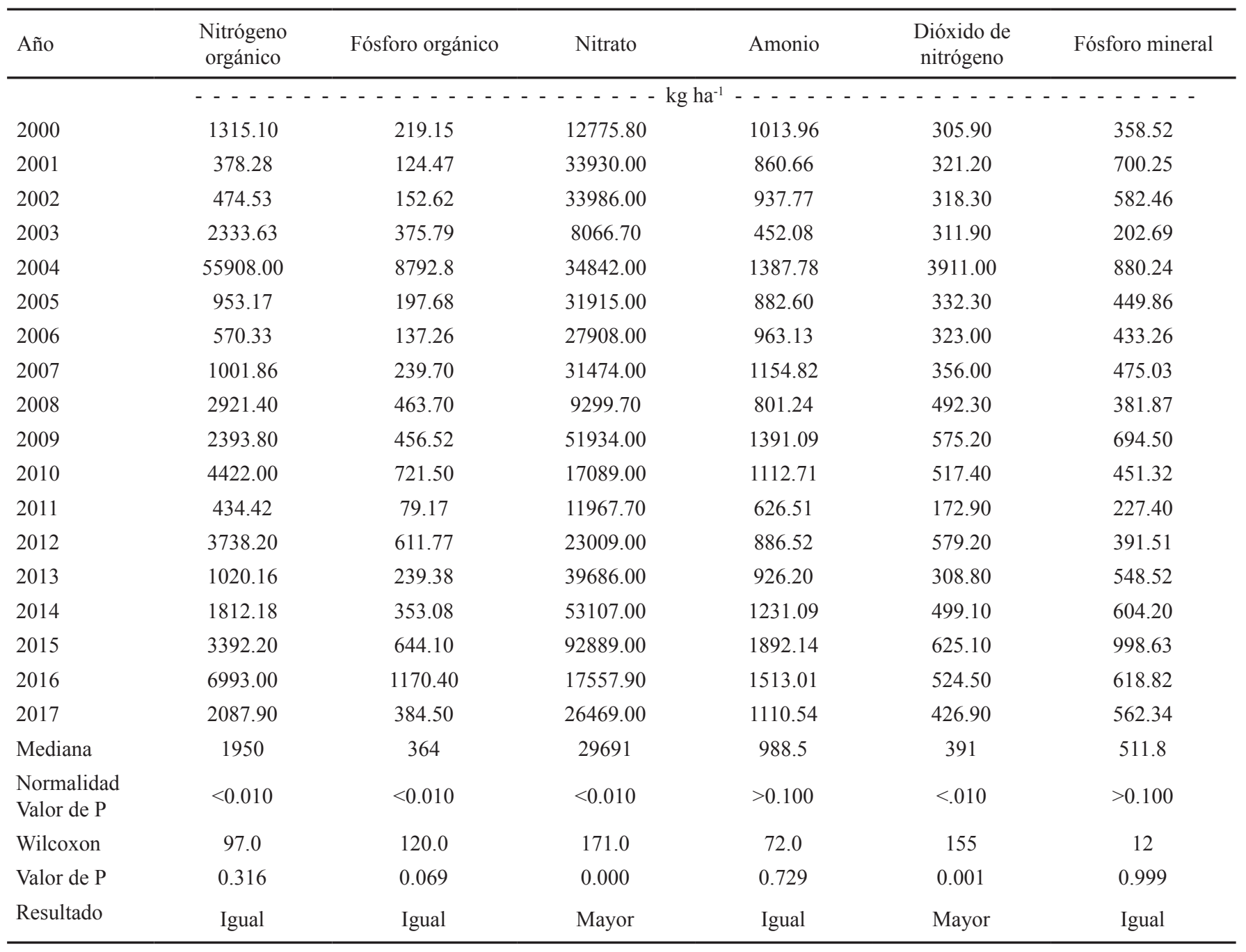

Fuente: Elaboración propia (2019). Source: Elaborated by the authors (2019).

La falta de estudios que señalen lo anterior, de acuerdo en lo establecido por Martínez-Yrizar et al. (2017) ha llevado al desconocimiento de las aportaciones de substancias como nitrógeno y fósforo dentro de los ecosistemas mexicanos. De igual manera esta implicación deberá establecer cambios potenciales en la mancha agrícola de la cuenca, e incluso sus afectaciones al entorno social.

\section{CONCLUSIONES}

- De acuerdo con los resultados obtenidos en la simulación realizada, se observan diferencias entre las aportaciones de contaminantes analizados por el modelo hidrológico SWAT, además de arrojar significancia estadística para dos de estos. Lo que comprueba que la actividad agrícola dentro de la cuenca hidrológica analizada genera repercusiones en los cuerpos de agua. El uso de Sistemas de Información Geográfica ayuda a la simulación de estas prácticas con las cuales se pueden parametrizar los efectos de su implementación a escala geográfica. Es necesario establecer los parámetros máximos permitidos para cada una de las substancias simuladas por el modelo hidrológico, así como la incorporación de indicadores de contaminación agrícola dentro del Catálogo Nacional de Indicadores del INEGI. 


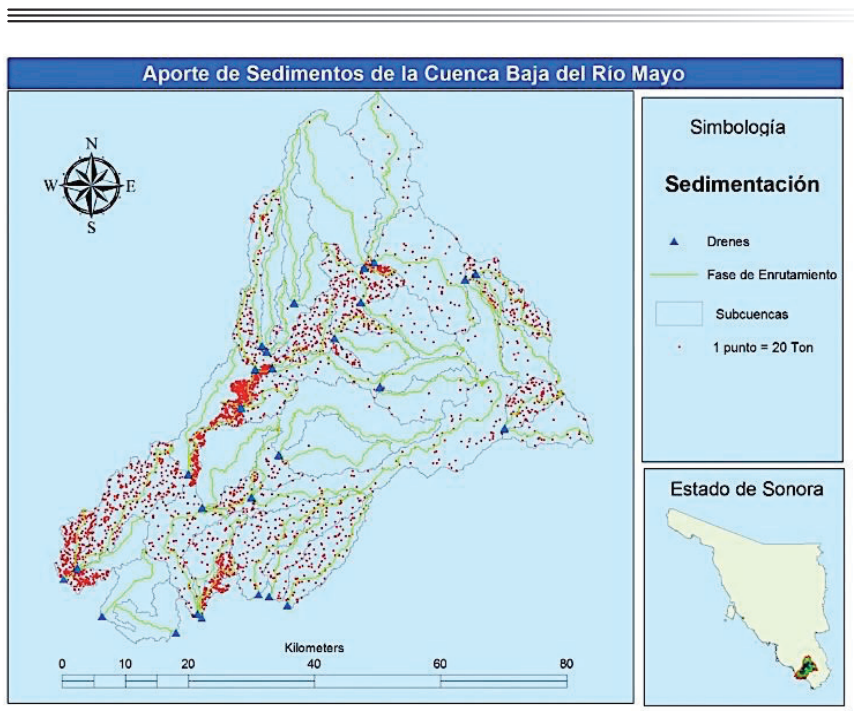

Figura 4. Aporte de sedimentos de la cuenca baja del río Mayo. Fuente: Elaboración propia (2019).

Figure 4. Sediment contribution from the lower basin of the Mayo River. Source: Elaborated by the authors (2019).

-Se debe de concluir que de acuerdo con el trabajo realizado destaca la pertinencia de realizar estudios a nivel de cuencas hidrológicas, que caractericen el comportamiento entre la interacción de las actividades antropogénicas con los componentes de los ecosistemas en la generación de contaminantes.

\section{LITERATURA CITADA}

Andrews, K. S., G. D. Williams, J. F. Samhouri, K. N. Marshall, V. Gertseva, and P. S. Levin. 2015. The legacy of a crowded ocean: indicators, status, and trends of anthropogenic pressures in the California Current ecosystem. Environ. Conserv. 139151. doi: https://doi.org/10.1017/S0376892914000277.

Babin, N. L., N. D. Mullendore, and L. S. Prokopy. 2016. Using social criteria to select watersheds for non-point source agricultural pollution abatement projects. Land Use Pol. 55: 327-333. doi: https://doi.org/10.1016/j.landusepol.2015.06.021.

CONABIO (Comisión Nacional para el Conocimiento y Uso de la Biodiversidad). 2016. Portal de geoinformación; Sistema Nacional de Información sobre Biodiversidad. http://www. conabio.gob.mx/informacion/gis/ (Consulta: mayo 30, 2018).

Cotler-Ávales, H., A. Galindo, I. González, R. Pineda y E. Ríos. 2013. Cuencas hidrográficas. Fundamentos y perspectivas para su manejo y gestión. SEMARNAT. México, D. F.

Galloway, J., A. Townsend, J. Erisman, M. Bekunda, Z. Cai, J. Freney, L. Martinelli, S. Seitzinger, and M. Sutton. 2008. Transformation of the nitrogen cycle: Recent trends, questions and potential solutions. Science 320: 889-892. doi: 10.1126/ science. 1136674 .

González Herrera, R. A., B. S. I. Albornoz Euán, I. A. Sánchez y Pinto y J. H. Osorio Rodríguez. 2018. El acuífero yucateco.
Análisis del riesgo de contaminación con apoyo de un sistema de información geográfica. Rev. Int. Contam. Amb. 34: 667-683. doi: 10.20937/RICA.2018.34.04.09.

Haro-Martínez, A. A. y I. C. Taddei. 2014. Sustentabilidad y economía: la controversia de la valoración ambiental. Econ. Soc. Territ. 14: 743-767.

Haro-Martínez, A. A., H. M. Arias-Rojo y I. C. Taddei. 2015. El valor de los servicios ambientales en la cuenca baja del río Mayo. Región Soc. 27: 31-59.

INEGI (Instituto Nacional de Estadística y Geografía). 2016. Datos de relieve. https://www.inegi.org.mx/app/geo2/ elevacionesmex/index.jsp (Consulta: mayo 20, 2018).

Large, S. I., G. Fay, K. D. Friedland, and J. S. Link. 2013. Defining trends and thresholds in responses of ecological indicators to fishing and environmental pressures. ICES J. Marine Sci. 70: 755-767. doi: https://doi.org/10.1093/icesjms/fst067.

Linder, H. L., J. K. Horne, and E. J. Ward. 2017. Modeling baseline conditions of ecological indicators: Marine renewable energy environmental monitoring. Ecol. Indicat. 83: 178-191. doi: https://doi.org/10.1016/j.ecolind.2017.07.015.

Liu, R., P. Zhang, X. Wang, Y. Chen, and Z. Shen. 2013. Assessment of effects of best management practices on agricultural non-point source pollution in Xiangxi River watershed. Agric. Water Manage. 117: 9-18. https://doi.org/10.1016/j. agwat.2012.10.018

Madroñero Palacios, S. M. y F. Jiménez Otárola. 2019. Manejo del recurso hídrico y estrategias de gestión integral en la microcuenca del río Mijitayo, Colombia. 2. Fuentes de contaminación y calidad del recurso hídrico. Recur. Nat. Amb. 56-57: 51-58.

Maharjan, G. R., M. Ruidisch, C. L. Shope, K. Choi, B. Huwe, S. J. Kim, J. Tenhunen, and S. Arnhold. 2016. Assessing the effectiveness of split fertilization and cover crop cultivation in order to conserve soil and water resources and improve crop productivity. Agric. Water Manage. 163: 305-318. https://doi. org/10.1016/j.agwat.2015.10.005.

Martínez-Yrizar, A., J. Álvarez-Sánchez y M. Maass. 2017. Análisis y perspectivas del estudio de los ecosistemas terrestres de México: Dinámica hidrológica y flujos de nitrógeno y fósforo. Rev. Mex. Biodiver. 88: 27-41. doi: https://doi.org/10.1016/j. rmb.2017.10.008.

Neitsch, S. L., J. G. Arnold, J. R. Kiniry, and J. R. Williams. 2011. Soil and water assessment tool theoretical documentation version 2009. Texas Water Resources Institute. Available electronically from http:/hdl.handle.net/1969.1/128050. College Station, TX, USA.

Oliveira, L. M., P. Maillard, and E. J. Andrade Pinto. 2017. Application of a land cover pollution index to model non-point pollution sources in a Brazilian watershed. CATENA 150: 124-132. doi: https://doi.org/10.1016/j.catena.2016.11.015.

Ouyang, W., X. Hao, L.Wang, Y. Xu, M. Tysklind, X. Gao, and C. Lin. 2019. Watershed diffuse pollution dynamics and response to land development assessment with riverine sediments. Sci. Total Environ. 659: 283-292. doi: https://doi.org/10.1016/j. scitotenv.2018.12.367.

Özcan, Z., O. Başkan, H. Düzgün, E. Kentel, and E. Alp. 2017. A pollution fate and transport model application in a semiarid region: Is some number better than no number? Sci. Total Environ. 595: 425-440. doi: https://doi.org/10.1016/j. scitotenv.2017.03.240. 
Reaney, S. M., E. B. Mackay, P. M. Haygarth, M. Fisher, A. Molineux, M. Potts, and C. McW. H. Benskin. 2019. Identifying critical source areas using multiple methods for effective diffuse pollution mitigation. J. Environ. Manage. 250: 109366. doi: https://doi.org/10.1016/j.jenvman.2019.109366.

Reid, W. V., H. A. Mooney, A. Cropper, D. Capistrano, S. R. Carpenter, K. Chopra, P. Dasgupta, T. Dietz, A. K. Duraiappah, R. Hassan, R. Kasperson, R. Leemans, R. M. May, Tony (A. J.) McMichael, P. Pingali, C. Samper, R. Scholes, R. T. Watson, A. H. Zakri, Z. Shidong, N. J. Ash, E. Bennett, P. Kumar, M. J. Lee, C. Raudsepp-Hearne, H. Simons, J. Thonell y M. B. Zurek. 2005. Evaluación de los Ecosistemas del Milenio. Informe de Síntesis. Millennium Ecosystem Assessment. ONU. New York, NY, USA.

Smith, L., A. Inman, X. Lai, H. Zhang, M. Fanqiao, Z. Jianbin, S. Burke, C. Rahn, G. Siciliano, P. M. Haygarth, J. Bellarby, and B. Surridge. 2017. Mitigation of diffuse water pollution from agriculture in England and China, and the scope for policy transfer. Land Use Policy 61: 208-219. doi: https://doi. org/10.1016/j.landusepol.2016.09.028.

SWAT (Soil and Water Assessment Tool). 2018. Global weather data for SWAT. Obtained from: https:/globalweather.tamu. edu. Texas \& A. M. University System. College Station, TX, USA.
Turner, J., H. J. Albrechtsen, M. Bonell, J. P. Duguet, B. Harris, R. Meckenstock, K. McGuire, R. Moussa, N. Peters, H. Richnow, B. Sherwood-Lollar, S. Uhlenbrook, and H. van Lanen. 2006. Future trends in transport and fate of diffuse contaminants in catchments, with special emphasis on stable isotope applications. Hydrol. Processes 20: 205-213. doi: https://doi. org/10.1002/hyp.6074.

Xiao, L., B. Xie, J. Liu, H. Zhang, G. Han, O. Wang, and F. Liu. 2017. Stimulation of long-term ammonium nitrogen deposition on methanogenesis by Methanocellaceae in a coastal wetland. Sci. Total Environ. 595: 337-343. doi: https:// doi.org/10.1016/j.scitotenv.2017.03.279.

Zhang, X., Y. Wu, and B. Gu. 2015. Urban rivers as hotspots of regional nitrogen pollution. Environ. Pollut. 205: 139-144. doi: https://doi.org/10.1016/j.envpol.2015.05.031.

Zhou, T., J. Wu, and S. Peng. 2012. Assessing the effects of landscape pattern on river water quality at multiple scales: a case study of the Dongjiang River watershed, China. Ecol. Indicat. 23: 166-175. doi: https://doi.org/10.1016/j.ecolind.2012.03.013. 